\title{
Footwear modifies coronal plane forefoot and sagittal plane hallux kinematics during stance phase of walking gait
}

\section{Chris Bishop , Gunther Paul \& Dominic Thewlis}

To cite this article: Chris Bishop , Gunther Paul \& Dominic Thewlis (2011) Footwear modifies coronal plane forefoot and sagittal plane hallux kinematics during stance phase of walking gait, Footwear Science, 3:sup1, S12-S13, DOI: 10.1080/19424280.2011.575839

To link to this article: http://dx.doi.org/10.1080/19424280.2011.575839

\section{曲 Published online: 30 Jun 2011.}

Submit your article to this journal $₫$

Џ Article views: 70

Q View related articles ๔ 


\section{References}

Mauch, M., et al., 2009. Ergonomics, 52 (8), 999-1008.
Schmeltzpfenning, T., et al., 2010. Proceedings 3rd AHFE, ISBN-13: 978-0-9796435-4-5.

\title{
Footwear modifies coronal plane forefoot and sagittal plane hallux kinematics during stance phase of walking gait
}

\author{
Chris Bishop*, Gunther Paul and Dominic Thewlis \\ University of South Australia, Adelaide, Australia
}

\section{Introduction}

Footwear is designed to reduce injury, and enhance performance. However, the effect footwear has on foot and ankle kinematics currently remains unknown. Acknowledging the need for improved understanding, multi-segment models of the foot-shoe complex need to be established to both describe and quantify the effect footwear has on the foot and ankle during stance phase of gait.

\section{Purpose of the Study}

The purpose of this study was to quantify how footwear alters the kinematics of the foot inside the shoe during stance phase of walking gait.

\section{Methods}

Fourteen participants, with a mean age of $21.8 \mathrm{yrs}$ ( \pm 3.5 years), height of $1.75 \mathrm{~m}( \pm 0.09 \mathrm{~m})$ and body mass of $71.0 \mathrm{~kg}( \pm 10.6 \mathrm{~kg})$ were recruited. In order to quantify the effect of footwear independent of technical design features, an ASICS Onitsuka Tiger-Mexico 66 shoe was used in this study. A newly developed marker set was implemented, which tracked shod and in-shoe kinematic of the foot and ankle. The marker set was applied in four experimental conditions during walking gait; barefoot, shod, modified shod and inshoe. To define in-shoe kinematics, $10 \mathrm{~mm}$ diameter were punched in the shoe upper, with custom tracking markers developed to mount a marker on a $25 \mathrm{~mm}$ wand through the shoe. These holes were cut as close to the original tracking markers as possible $(10-20 \mathrm{~mm})$.

The calibration markers were uniform to all footwear conditions. The modified shod condition was defined as markers placed on the shoe once holes had been cut in the shoe upper. This condition was used to determine the effect that cutting holes in the shoe upper. The complete in-shoe marker set is presented in Figure 1. Participants completed five trials in each experimental condition during one session. Kinematic data were captured using a 12-camera VICON MX40 motion capture system (Vicon Motion Systems Ltd, Oxford UK) at $100 \mathrm{~Hz}$ and processed in Visual3D (C-Motion Inc, USA). The kinematic data were lowpass filtered with a fourth-order Butterworth filter at $7 \mathrm{~Hz}$. Data were extracted for each segment in each experimental condition at initial contact, loading response $(15 \%$ stance $)$, midstance $(50 \%$ stance $)$ and propulsion ( $95 \%$ stance).

\section{Results}

Walking speed was not significantly different between data collection sessions $(p>0.05)$. No significant differences were detected at any event in the first half of stance. At midstance, the in-shoe forefoot was $8.3^{\circ}$ significantly more plantarflexed than the barefoot

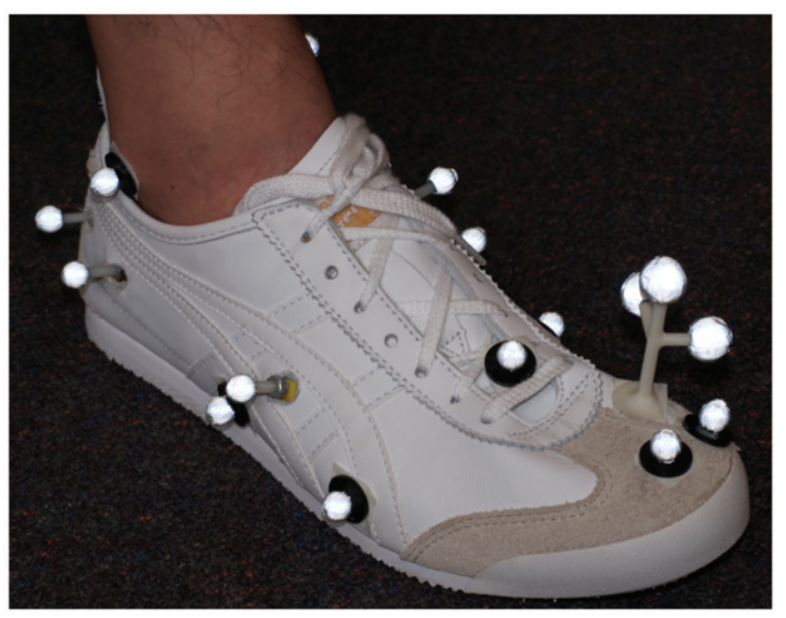

Figure 1. Marker set.

*Corresponding author. Email: biscm002@mymail.unisa.edu.au 
Table 1. Foot-shoe complex marker set.

\begin{tabular}{lll}
\hline Segment & \multicolumn{1}{c}{ Calibration markers } & \multicolumn{1}{c}{ Tracking markers } \\
\hline Shank & R Lat Fem Epicondyle & Cluster (four markers) on \\
& R Med Fem Epicondyle & distal 1/3 of segment \\
R Lateral Malleolus & \\
R Medial Malleolus & \\
Hindfoot & R Lateral Malleolus & R Lat Calc \\
& R Medial Malleolus & R Post-lat calc \\
& R Styloid Process & R Post-med calc \\
R Navicular Tuberosity & R Med Calc \\
Forefoot & R Styloid Process & R med 1st Met Shaft (proximal) \\
& R Navicular Tuberosity & R lat 4th met shaft (mid) \\
R 1st Met Head (medial) & \\
R 5th Met Head (lateral) & Hallux trihedron \\
R 1st Met Head (medial) & \\
R 5th Met Head (lateral) & \\
R Hallux (apex) & \\
R 2nd Toe (apex) & \\
\hline
\end{tabular}

condition $(p<0.001)$. The in-shoe forefoot was $3.4^{\circ}$ significantly more abducted than the shod condition $(p=0.023)$. At propulsion, the in-shoe hallux angle was significantly less dorsiflexed (mean difference $10.6^{\circ}$ ) than the barefoot condition $(p<0.001)$.

Modification of footwear significantly altered the shod kinematics of the hallux in both the coronal (Mean Difference (MD) - 6.6,$p=0.003$ ) and transverse planes $\left(\mathrm{MD}-9.4^{\circ}, p<0.001\right)$ at midstance. During propulsion, the modification of footwear significantly altered shod kinematics of the hallux in the sagittal $\left(\mathrm{MD}-8.1^{\circ}, p=0.002\right)$ and transverse planes $\left(\mathrm{MD}-10.3^{\circ}, p<0.001\right)$.

\section{Discussion and conclusion}

This study demonstrates that footwear has significant effects on sagittal and coronal plane kinematics of the foot in the shoe. Important in the context of these significant findings, the modification of footwear did not significantly alter the shod kinematics of the hindfoot or forefoot during stance. The current marker set is limited in its ability to track the kinematics of shod hallux once modification of the shoe has occurred.

This paper presents a method for describing the inshoe kinematics of the foot and ankle during stance phase of walking gait. In conclusion, the model proposed provides a realistic representation of the effect footwear has on multi-segment foot and ankle kinematics.

\section{References}

Cappozzo, A., 1995. Clin Biomech, 10 (4), 171-178.

Grood, E and Suntay, W., 1983. Transactions of the ASME, 105, 136-144.

\title{
The development of a multi-segment kinematic model of footwear
}

\author{
Chris Bishop*, Gunther Paul, Hayley Uden and Dominic Thewlis
}

University of South Australia, Adelaide, Australia

\section{Introduction}

In gait analysis, both shoe mounted and skin mounted markers have been used to quantify the movement of the foot inside the shoe (Reinschmidt et al. 1992). However, these models have not been demonstrated as reliable or accurate in shod conditions.

*Corresponding author. Email: biscm002@mymail.unisa.edu.au 